\title{
Publisher Correction: Letter perception emerges from unsupervised deep learning and recycling of natural image features
}

Alberto Testolin, Ivilin Stoianov and Marco Zorzi

Correction to: Nature Human Behaviour https://doi.org/10.1038/s41562-017-0186-2 (2017); published online 21 August 2017.

In the version of this Letter originally published, in the sentence beginning "Written symbols are culture specific..., in the second example, ' $\Phi$ ' was used instead of 'F'; it should have read '(for example, $\mathcal{F}$ versus $\mathbf{F}$ )'. This has now been corrected in all versions of the Letter.

Published online: 2 November 2017

https://doi.org/10.1038/s41562-017-0253-8 